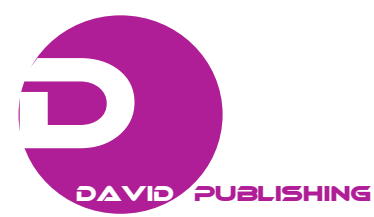

\title{
Synthesis and Study of Heteronuclear Citrates
}

\author{
Nani Bidzina Zhorzholiani*, Iamze Alim Beshkenadze, Maia Avtandil Gogaladze and Givi Tengiz Begheluri \\ Petre Melikishvili Institute of Physical and Organic Chemistry, Ivane Javakhishvili Tbilisi State University, Tbilisi 0186, Georgia
}

Received: March 08, 2014 / Accepted: April 01, 2014 / Published: April 25, 2014.

\begin{abstract}
Terms of synthesis were determined for creation of new generation premixes and for their testing in experiments. Heteronuclear chelate citrates of general formula: $\mathrm{M}_{2}^{\mathrm{I}} \mathrm{M}^{\mathrm{II}} \mathrm{L}_{2} \cdot n \mathrm{H}_{2} \mathrm{O}$ (where, $\mathrm{M}^{\mathrm{I}}=\mathrm{Zn}, \mathrm{Co}, \mathrm{Fe}, \mathrm{Mn}, \mathrm{Cu} ; \mathrm{M}^{\mathrm{II}}=\mathrm{Mn}, \mathrm{Zn}, \mathrm{Co}, \mathrm{Cu} ; n=\mathrm{O} / 4$ ) were synthesized. Identity and composition of synthesized compounds were defined by microelemental analysis, determination of melting temperature and X-ray diffraction analysis. X-ray diffraction method was used also to define crystallinity of the compounds and their citric acid $\left(\mathrm{H}_{4} \mathrm{~L}\right)$ component. X-ray amorphous and iso-structural orders were also revealed.
\end{abstract}

Key words: Bio-metals, chelate compounds, microelemental analysis, X-ray diffraction analysis.

\section{Introduction}

Even the most primitive forms of live organisms are unable to provide vital processes without participation of bio-metals. In optimal doses they are able to affect actively growth and development, propagation, productivity, resistance of organisms to diseases. Investigations of researchers prove that bio-metals play active role in the physiological and biological processes going on in poultry and animals. Up to 75 chemical elements are found in their organisms and namely this conditioned the interest shown to the so-called "vital metals" by wide specter of researchers. We considered five microelements ( $\mathrm{Zn}, \mathrm{Co}, \mathrm{Fe}, \mathrm{Mn}$, $\mathrm{Cu}$ ), because these microelements belong to a number of very critical and essential for life elements that animal organisms have. They play an important role in a water and organic compounds metabolism, absorbtion and utilization of nutrients. They generateoptimal conditions for the normal function of many organs including cardiac muscule and nervous system [1-4]. Their role and significance are exemplified by the fact that one of the main factors which defines low quantity and quality indices of

"Corresponding author: Nani Bidzina Zhorzholiani, Association Professor, research fields: inorganic and coordination chemistry. E-mail: nanizhorzholi@gmail.com. foodstuff is the deficiency of bio-metals in plants, soil, as well as in agricultural animals and poultry. Decisive role in the resolution of this problem is attributed to the provision of live organisms with optimal quantity and ratio of microelements. Since bio-metals in live organisms fulfill their functions in the form of chelates, at filling up of deficit in bio-metals in the form of chelates, we observe sharp increase in their biological activity [5-8].

\section{Materials and Methods}

For synthesis, we used the following original compounds: $\mathrm{ZnO}, \quad \mathrm{MnCO}_{3}, \quad \mathrm{CoCO}_{3} \mathrm{Co}(\mathrm{OH})_{2}$, $\mathrm{CuCO}_{3} \mathrm{Cu}(\mathrm{OH})_{2}, \mathrm{Fe}\left(\mathrm{CH}_{3} \mathrm{COO}\right)_{2} \cdot 4 \mathrm{H}_{2} \mathrm{O}$, and important for vital processess oxyacid-citric acid $\left(\mathrm{H}_{4} \mathrm{~L}\right)$. Composition of compounds and their identity were defined by well-known methods: microelemental analysis (CHN Analyser, Labertherm GmbH). Purity was determined by melting temperature measuring (Dynalon SMP10) and X-ray diffraction method on DPOH-3Mtype diffractometer, at copper anode emission. The same method was used to define their crystallinity, and X-ray amorphous and iso-structural orders were also revealed.

\section{Results}

Investigations are continued at the Laboratory of 
Problems Agrarian Chemistry for creation of new generation premixes and for their testing in experiments [9-17]. With this in view heteronuclear chelate citrates of the following general formula:

$\mathrm{M}_{2}^{\mathrm{I}} \cdot \mathrm{M}^{\mathrm{II}} \cdot \mathrm{L}_{2} \cdot n \mathrm{H}_{2} \mathrm{O}$ (where, $\mathrm{M}^{\mathrm{I}}=\mathrm{Zn}, \mathrm{Co}, \mathrm{Fe}, \mathrm{Mn}, \mathrm{Cu}$; $\left.\mathrm{M}^{\mathrm{II}}=\mathrm{Mn}, \mathrm{Zn}, \mathrm{Co}, \mathrm{Cu} ; n=\mathrm{O} / 4\right)$ were created. To obtain these citrates, we take a mix: (1) $\mathrm{ZnO}: \mathrm{MnbCO}_{3}$, (2) $\mathrm{CoCO}_{3} \mathrm{Co}(\mathrm{OH})_{2}: \mathrm{ZnO}$, (3) $\mathrm{CuCO}_{3} \mathrm{Cu}(\mathrm{OH})_{2}$ : $\mathrm{CoCO}_{3} \mathrm{Co}(\mathrm{OH})_{2}$, (4) $\mathrm{CoCO}_{3} \mathrm{Co}(\mathrm{OH})_{2}: \mathrm{MnCO}_{3}$, (5) $\mathrm{CoCO}_{3} \mathrm{Co}(\mathrm{OH})_{2}: \mathrm{CuCO}_{3} \mathrm{Cu}(\mathrm{OH})_{2}$,

$\mathrm{Fe}\left(\mathrm{CH}_{3} \mathrm{COO}\right)_{2} \cdot 4 \mathrm{H}_{2} \mathrm{O}: \mathrm{MnCO}_{3}$,

$\mathrm{Fe}\left(\mathrm{CH}_{3} \mathrm{COO}\right)_{2} \cdot 4 \mathrm{H}_{2} \mathrm{O}: \mathrm{ZnO}$,

$\mathrm{Fe}\left(\mathrm{CH}_{3} \mathrm{COO}\right)_{2} \cdot 4 \mathrm{H}_{2} \mathrm{O}: \mathrm{CoCO}_{3} \mathrm{Co}(\mathrm{OH})_{2}$ and (9) $\mathrm{MnCO}_{3}: \mathrm{CuCO}_{3} \mathrm{Cu}(\mathrm{OH})_{2}$ at $2: 1$ molar ration on porcelain cup, crush intensely till it is reduced to fine dispersion condition, then we add citric acid dissolved in minimal volume of hot water. We observe intense isolation of $\mathrm{CO}_{2}$ and changes of color. The obtained solutions are left at room temperature till the next day, are filtered and the residue after its washing by ether is dried on air.

Identity and composition of synthesized chelate composites: $\quad \mathrm{Zn}_{2} \cdot \mathrm{Mn} \cdot \mathrm{L}_{2} \cdot 2 \mathrm{H}_{2} \mathrm{O}, \quad \mathrm{Co}_{2} \cdot \mathrm{Zn} \cdot \mathrm{L}_{2}$, $\mathrm{Cu}_{2} \cdot \mathrm{Co} \cdot \mathrm{L}_{2} \cdot \mathrm{H}_{2} \mathrm{O}, \quad \mathrm{Fe}_{2} \cdot \mathrm{Mn} \cdot \mathrm{L}_{2} \cdot 2 \mathrm{H}_{2} \mathrm{O}, \mathrm{Co}_{2} \cdot \mathrm{Mn} \cdot \mathrm{L}_{2} \cdot 4 \mathrm{H}_{2} \mathrm{O}$, $\mathrm{Co}_{2} \mathrm{CuL}_{2} \cdot 4 \mathrm{H}_{2} \mathrm{O}, \mathrm{Mn}_{2} \mathrm{CuL}_{2} \cdot 4 \mathrm{H}_{2} \mathrm{O}, \mathrm{Fe}_{2} \mathrm{ZnL}_{2} \cdot 2 \mathrm{H}_{2} \mathrm{O}$ and $\mathrm{Fe}_{2} \mathrm{CoL}_{2} \cdot 4 \mathrm{H}_{2} \mathrm{O}$ were defined by microelemental analysis (Table 1), by melting temperature detection (Table 2), by X-ray diffraction method (Table 3).

We also defined their crystallinity and revealed $\mathrm{X}$-ray amorphous and iso-structural orders by $\mathrm{X}$-ray diffraction analysis.

Table 3 represents the results of relative intensities $\left(I / I_{0}\right)$, reflection angle $(2 \theta)$, inter-plane distances $(d)$ obtained by deciphering of X-ray diffraction patterns of the composites.

\section{Discussions}

X-ray diffraction studies did not reveal presence of starting compounds in the samples in the form of separate phase. Therefore, we can conclude that

Table 1 Results of microelemental analysis of chelates.

\begin{tabular}{|c|c|c|c|c|c|c|c|c|c|}
\hline \multirow{2}{*}{ \# } & \multirow{2}{*}{ Formula } & \multicolumn{4}{|c|}{ Practical (\%) } & \multicolumn{4}{|c|}{ Theoretical (\%) } \\
\hline & & $\mathrm{M}^{\mathrm{I}}$ & $\mathrm{M}^{\mathrm{II}}$ & $\mathrm{C}$ & $\mathrm{H}$ & $\mathrm{M}^{\mathrm{I}}$ & $\mathrm{M}^{\mathrm{II}}$ & $\mathrm{C}$ & $\mathrm{H}$ \\
\hline 1 & $\mathrm{Zn}_{2} \cdot \mathrm{Mn} \cdot \mathrm{L}_{2} \cdot 4 \mathrm{H}_{2} \mathrm{O}$ & 20.94 & 9.05 & 24.03 & 2.15 & 21.69 & 9.17 & 24.00 & 2.34 \\
\hline 2 & $\mathrm{Co}_{2} \cdot \mathrm{Zn} \cdot \mathrm{L}_{2}$ & 20.87 & 10.72 & 25.09 & 2.17 & 21.01 & 11.58 & 25.66 & 2.49 \\
\hline 3 & $\mathrm{Cu}_{2} \cdot \mathrm{Co} \cdot \mathrm{L}_{2} \cdot \mathrm{H}_{2} \mathrm{O}$ & 22.11 & 9.96 & 24.45 & 1.91 & 21.81 & 10.12 & 24.73 & 2.06 \\
\hline 4 & $\mathrm{Co}_{2} \cdot \mathrm{Mn} \cdot \mathrm{L}_{2} \cdot 4 \mathrm{H}_{2} \mathrm{O}$ & 19.03 & 8.73 & 20.96 & 2.72 & 18.91 & 8.83 & 21.11 & 2.89 \\
\hline 5 & $\mathrm{Co}_{2} \cdot \mathrm{Cu} \cdot \mathrm{L}_{2} \cdot 4 \mathrm{H}_{2} \mathrm{O}$ & 18.21 & 9.79 & 22.75 & 2.63 & 18.66 & 10.05 & 22.79 & 2.85 \\
\hline 6 & $\mathrm{Fe}_{2} \cdot \mathrm{Mn} \cdot \mathrm{L}_{2} \cdot 2 \mathrm{H}_{2} \mathrm{O}$ & 19.33 & 9.14 & 24.62 & 2.15 & 19.27 & 9.46 & 24.77 & 2.41 \\
\hline 7 & $\mathrm{Fe}_{2} \cdot \mathrm{Zn} \cdot \mathrm{L}_{2} \cdot 2 \mathrm{H}_{2} \mathrm{O}$ & 19.15 & 11.13 & 24.79 & 2.23 & 18.94 & 10.99 & 24.35 & 2.37 \\
\hline 8 & $\mathrm{Fe}_{2} \cdot \mathrm{Co} \cdot \mathrm{L}_{2} \cdot 4 \mathrm{H}_{2} \mathrm{O}$ & 17.78 & 8.93 & 22.98 & 2.65 & 18.03 & 9.49 & 23.18 & 2.89 \\
\hline 9 & $\mathrm{Mn}_{2} \cdot \mathrm{Cu} \cdot \mathrm{L}_{2} \cdot 4 \mathrm{H}_{2} \mathrm{O}$ & 17.17 & 10.09 & 22.97 & 2.77 & 17.63 & 10.18 & 23.08 & 2.89 \\
\hline
\end{tabular}

Table 2 Results of measurements of composites melting temperatures.

\begin{tabular}{llll}
\hline$\#$ & Formula & Melting, $\mathrm{T}\left({ }^{\circ} \mathrm{C}\right)$ & Color \\
\hline 1 & $\mathrm{Zn}_{2} \cdot \mathrm{Mn} \cdot \mathrm{L}_{2} \cdot 4 \mathrm{H}_{2} \mathrm{O}$ & $>286$ & White \\
2 & $\mathrm{Co}_{2} \cdot \mathrm{Zn} \cdot \mathrm{L}_{2}$ & $>286$ & Lilac \\
3 & $\mathrm{Cu}_{2} \cdot \mathrm{Co} \cdot \mathrm{L}_{2} \cdot \mathrm{H}_{2} \mathrm{O}$ & $>286$ & Dark light-blue \\
4 & $\mathrm{Co}_{2} \cdot \mathrm{Mn} \cdot \mathrm{L}_{2} \cdot 4 \mathrm{H}_{2} \mathrm{O}$ & $>286$ & Grey-lilac \\
5 & $\mathrm{Co}_{2} \cdot \mathrm{Cu} \cdot \mathrm{L}_{2} \cdot 4 \mathrm{H}_{2} \mathrm{O}$ & $>286$ & Dark lilac \\
6 & $\mathrm{Fe}_{2} \cdot \mathrm{Mn} \cdot \mathrm{L}_{2} \cdot 2 \mathrm{H}_{2} \mathrm{O}$ & $>286$ & Mustard yellow \\
7 & $\mathrm{Fe}_{2} \cdot \mathrm{Zn} \cdot \mathrm{L}_{2} \cdot 2 \mathrm{H}_{2} \mathrm{O}$ & $>286$ & Light brown \\
8 & $\mathrm{Fe}_{2} \cdot \mathrm{Co} \cdot \mathrm{L}_{2} \cdot 4 \mathrm{H}_{2} \mathrm{O}$ & $>286$ & Grey-brown \\
9 & $\mathrm{Mn}_{2} \cdot \mathrm{Cu} \cdot \mathrm{L}_{2} \cdot 4 \mathrm{H}_{2} \mathrm{O}$ & $>286$ & Light green \\
\hline
\end{tabular}


Table 3 Results of X-ray diffraction analysis of chelate composites and citric acid.

\begin{tabular}{|c|c|c|c|c|c|c|c|c|c|c|c|}
\hline \multicolumn{3}{|c|}{$\mathrm{H}_{4} \mathrm{~L}$} & \multicolumn{3}{|c|}{ 1. $\mathrm{Zn}_{2} \cdot \mathrm{Mn} \cdot \mathrm{L}_{2} \cdot 4 \mathrm{H}_{2} \mathrm{O}$} & \multicolumn{3}{|c|}{ 2. $\mathrm{Co}_{2} \cdot \mathrm{Zn} \cdot \mathrm{L}_{2}$} & \multicolumn{3}{|c|}{ 3. $\mathrm{Cu}_{2} \cdot \mathrm{Co}_{2} \mathrm{~L}_{2} \cdot \mathrm{H}_{2} \mathrm{O}$} \\
\hline $2 \theta$ & $d$ & $J / J_{0}$ & $2 \theta$ & $d$ & $J / J_{0}$ & $2 \theta$ & $d$ & $J / J_{0}$ & $2 \theta$ & $d$ & $J / J_{0}$ \\
\hline 11.5 & 0.3 & 2.5 & 10.66 & 8.301 & 0.0608 & 8.500 & 8.152 & 1.1696 & 10.89 & 8.120 & 0.2350 \\
\hline 15.2 & 4.5 & 37.5 & 11.49 & 7.699 & 0.0043 & 11.37 & 7.782 & 0.0393 & 12.31 & 7.187 & 0.1800 \\
\hline 17.0 & 1.6 & 13.3 & 11.93 & 7.419 & 0.0530 & 12.27 & 7.214 & 0.2606 & 13.30 & 6.656 & 0.1033 \\
\hline 18.3 & 6.5 & 54.2 & 12.09 & 7.323 & 0.2286 & 13.32 & 6.645 & 0.1818 & 14.53 & 6.096 & 0.0100 \\
\hline 19.0 & 0.4 & 3.3 & 13.06 & 6.778 & 0.0156 & 15.72 & 5.636 & 0.0909 & 15.81 & 5.605 & 1.0000 \\
\hline 22.3 & 4.5 & 37.5 & 15.52 & 5.711 & 0.0486 & 15.83 & 5.599 & 0.2545 & 17.20 & 5.157 & 0.0866 \\
\hline 23.1 & 12.0 & 100.0 & 15.50 & 5.717 & 0.0660 & 16.66 & 5.322 & 0.0303 & 18.41 & 4.820 & 0.0533 \\
\hline 23.8 & 0.5 & 4.2 & 16.20 & 5.473 & 0.0173 & 17.23 & 5.147 & 0.0787 & 19.39 & 4.577 & 0.0683 \\
\hline 24.5 & 1.0 & 8.3 & 16.93 & 5.238 & 0.0269 & 18.47 & 4.803 & 0.0484 & 19.86 & 4.470 & 0.2166 \\
\hline 27.1 & 0.5 & 4.2 & 18.23 & 4.865 & 0.0026 & 20.03 & 4.434 & 1.0000 & 21.77 & 4.083 & 0.1316 \\
\hline 28.8 & 0.4 & 3.3 & 19.38 & 4.579 & 0.0382 & 20.34 & 4.367 & 0.3454 & 22.82 & 3.896 & 0.0350 \\
\hline 29.4 & 2.5 & 20.8 & 19.8 & 4.484 & 1.0000 & 20.67 & 4.298 & 0.0636 & 23.17 & 3.839 & 0.1533 \\
\hline 30.8 & 1.5 & 12.5 & 20.13 & 4.411 & 0.0376 & 21.70 & 4.095 & 0.0757 & 23.72 & 3.751 & 0.0933 \\
\hline 32.7 & 1.5 & 12.5 & 20.57 & 4.318 & 0.0339 & 21.75 & 4.085 & 0.1151 & 24.66 & 3.611 & 0.0816 \\
\hline 33.5 & 0.8 & 6.7 & 21.39 & 4.155 & 0.0921 & 22.36 & 3.976 & 0.0515 & 25.44 & 3.502 & 0.1183 \\
\hline 37.0 & 1.0 & 8.3 & 22.78 & 3.904 & 0.0269 & 22.90 & 3.881 & 0.1181 & 26.82 & 3.373 & 0.2483 \\
\hline 41.9 & 0.5 & 4.2 & 23.51 & 3.784 & 0.0113 & 23.81 & 3.737 & 0.0818 & 28.15 & 3.170 & 0.0433 \\
\hline 45.8 & 0.5 & 4.2 & 24.09 & 3.695 & 0.1052 & 24.31 & 3.661 & 0.0575 & 28.97 & 3.082 & 0.1216 \\
\hline \multirow[t]{27}{*}{56.8} & 0.5 & 4.2 & 24.4 & 3.648 & 0.0113 & 24.69 & 3.606 & 0.0303 & 30.44 & 2.937 & 0.0600 \\
\hline & & & 24.62 & 3.615 & 0.0147 & 26.54 & 3.358 & 0.2696 & 30.90 & 2.893 & 0.1316 \\
\hline & & & 26.07 & 3.418 & 0.0400 & 28.79 & 3.101 & 0.1242 & 31.81 & 2.813 & 0.0650 \\
\hline & & & 28.58 & 3.123 & 0.0800 & 29.41 & 3.035 & 0.0151 & 33.93 & 2.642 & 0.1050 \\
\hline & & & 29.24 & 3.056 & 0.0756 & 29.99 & 2.979 & 0.0878 & 35.49 & 2.529 & 0.0516 \\
\hline & & & 29.76 & 3.002 & 0.0939 & 31.07 & 2.879 & 0.2424 & 37.31 & 2.410 & 0.0350 \\
\hline & & & 30.32 & 2.948 & 0.0182 & 31.89 & 2.806 & 0.1515 & 40.36 & 2.235 & 0.0466 \\
\hline & & & 31.36 & 2.852 & 0.0313 & 32.43 & 2.761 & 0.0575 & & & \\
\hline & & & 32.25 & 2.775 & 0.0521 & 34.37 & 2.609 & 0.0424 & & & \\
\hline & & & 34.88 & 2.572 & 0.0252 & 35.87 & 2.503 & 0.0454 & & & \\
\hline & & & 35.64 & 2.512 & 0.0800 & 38.56 & 2.335 & 0.0181 & & & \\
\hline & & & 37.34 & 2.408 & 0.0443 & 40.26 & 2.240 & 0.0545 & & & \\
\hline & & & 38.77 & 2.323 & 0.0243 & 40.58 & 2.223 & 0.0424 & & & \\
\hline & & & 40.23 & 2.242 & 0.0104 & 42.22 & 2.190 & 0.0606 & & & \\
\hline & & & 40.39 & 2.233 & 0.0260 & & & & & & \\
\hline & & & 41.05 & 2.199 & 0.0330 & & & & & & \\
\hline & & & 42.91 & 2.107 & 0.0252 & & & & & & \\
\hline & & & 43.56 & 2.078 & 0.0269 & & & & & & \\
\hline & & & 43.70 & 2.072 & 0.0339 & & & & & & \\
\hline & & & 44.64 & 2.030 & 0.0313 & & & & & & \\
\hline & & & 45.49 & 1.994 & 0.0034 & & & & & & \\
\hline & & & 46.10 & 1.969 & 0.0243 & & & & & & \\
\hline & & & 46.19 & 1.965 & 0.0078 & & & & & & \\
\hline & & & 47.41 & 1.918 & 0.0365 & & & & & & \\
\hline & & & 48.15 & 1.890 & 0.0313 & & & & & & \\
\hline & & & 48.07 & 1.893 & 0.0495 & & & & & & \\
\hline & & & 49.2 & 1.852 & 0.0269 & & & & & & \\
\hline
\end{tabular}


Table 3 continued

\begin{tabular}{|c|c|c|c|c|c|c|c|c|c|c|c|}
\hline \multicolumn{3}{|c|}{ 4. $\mathrm{Co}_{2} \cdot \mathrm{Mn} \cdot \mathrm{L}_{2} \cdot 4 \mathrm{H}_{2} \mathrm{O}$} & \multicolumn{3}{|c|}{ 5. $\mathrm{Co}_{2} \cdot \mathrm{Cu} \cdot \mathrm{L}_{2} \cdot 4 \mathrm{H}_{2} \mathrm{O}$} & \multicolumn{3}{|c|}{ 6. $\mathrm{Fe}_{2} \cdot \mathrm{Zn} \cdot \mathrm{L}_{2} \cdot 2 \mathrm{H}_{2} \mathrm{O}$} & \multicolumn{3}{|c|}{ 7. $\mathrm{Mn}_{2} \cdot \mathrm{Cu} \cdot \mathrm{L}_{2} \cdot 4 \mathrm{H}_{2} \mathrm{O}$} \\
\hline $2 \theta$ & $d$ & $J / J_{0}$ & $2 \theta$ & $d$ & $J / J_{0}$ & $2 \theta$ & $d$ & $J / J_{0}$ & $2 \theta$ & $d$ & $J / J_{0}$ \\
\hline 10.70 & 8.267 & 0.0636 & 9.800 & 9.024 & 0.0645 & 6.67 & 13.247 & 1.0000 & 9.02 & 9.804 & 0.3888 \\
\hline 11.75 & 7.532 & 0.0454 & 10.84 & 8.160 & 0.1612 & 7.18 & 12.295 & 0.5714 & 10.77 & 8.213 & 0.5000 \\
\hline 13.17 & 6.725 & 0.0606 & 12.28 & 7.206 & 0.1612 & 8.44 & 10.466 & 0.1428 & 12.30 & 7.196 & 0.1611 \\
\hline 14.27 & 6.208 & 1.0000 & 15.49 & 5.719 & 0.0645 & 8.86 & 9.978 & 0.2857 & 13.10 & 6.760 & 0.2388 \\
\hline 14.84 & 5.968 & 0.0666 & 15.77 & 5.619 & 1.0000 & 10.87 & 8.133 & 0.6642 & 13.70 & 6.461 & 0.1111 \\
\hline 15.61 & 5.677 & 0.0909 & 17.07 & 5.193 & 0.0645 & 13.41 & 6.601 & 0.4285 & 13.74 & 6.445 & 0.0916 \\
\hline 17.02 & 5.210 & 0.0303 & 18.30 & 4.847 & 0.0967 & 14.34 & 6.175 & 0.2000 & 14.57 & 6.079 & 0.2500 \\
\hline 17.83 & 4.974 & 0.0939 & 19.77 & 4.488 & 0.1612 & 14.96 & 5.920 & 0.3214 & 15.58 & 5.688 & 0.7138 \\
\hline 18.39 & 4.824 & 0.4121 & 23.12 & 3.845 & 0.2580 & 17.01 & 5.211 & 0.2000 & 16.79 & 5.279 & 0.0722 \\
\hline 18.70 & 4.744 & 0.2303 & 23.28 & 3.820 & 0.1459 & 17.98 & 4.933 & 0.2857 & 16.93 & 5.236 & 0.2666 \\
\hline 20.42 & 4.348 & 0.3333 & 24.62 & 3.616 & 0.0967 & 19.49 & 4.553 & 0.1785 & 18.15 & 4.888 & 0.2500 \\
\hline 21.50 & 4.133 & 0.1333 & 25.36 & 3.511 & 0.1451 & 19.99 & 4.440 & 0.5000 & 18.22 & 4.870 & 0.1222 \\
\hline 21.61 & 4.110 & 0.3272 & 26.25 & 3.394 & 0.0645 & 20.63 & 4.303 & 0.1428 & 19.64 & 4.520 & 0.1611 \\
\hline 23.20 & 3.833 & 0.2303 & 27.48 & 3.245 & 0.0032 & 21.66 & 4.101 & 0.1785 & 20.21 & 4.393 & 0.1027 \\
\hline 23.59 & 3.772 & 0.1939 & 28.31 & 3.151 & 0.0516 & 22.46 & 3.958 & 0.1785 & 21.43 & 4.147 & 0.3055 \\
\hline 23.90 & 3.723 & 0.1393 & 29.30 & 3.047 & 0.0258 & 23.35 & 3.808 & 0.1428 & 21.83 & 4.072 & 0.2146 \\
\hline 24.65 & 3.612 & 0.0575 & 31.00 & 2.884 & 0.1290 & 26.99 & 3.303 & 0.1428 & 22.47 & 3.957 & 0.0833 \\
\hline 25.90 & 3.440 & 0.1606 & 31.49 & 2.840 & 0.0870 & 27.11 & 3.288 & 0.1785 & 23.44 & 3.795 & 0.1500 \\
\hline 26.96 & 3.307 & 0.2303 & 34.5 & 2.599 & 0.0645 & 30.77 & 2.906 & 0.1214 & 26.01 & 3.426 & 0.5000 \\
\hline 27.74 & 3.216 & 0.0606 & 37.41 & 2.404 & 0.0645 & 40.59 & 2.222 & 0.2142 & 28.77 & 3.103 & 0.1666 \\
\hline 28.70 & 3.111 & 0.1666 & 42.00 & 2.120 & 0.0419 & 46.04 & 1.971 & 0.1214 & 30.24 & 2.955 & 0.4166 \\
\hline 29.98 & 2.980 & 0.1515 & & & & 48.95 & 1.861 & 0.1428 & 31.59 & 2.832 & 0.2547 \\
\hline 31.74 & 2.819 & 0.1000 & & & & 55.10 & 1.666 & 0.1571 & 31.70 & 2.823 & 0.0962 \\
\hline 32.64 & 2.795 & 0.1763 & & & & & & & 33.10 & 2.706 & 0.1166 \\
\hline 33.91 & 2.662 & 0.1536 & & & & & & & 33.56 & 2.671 & 0.1611 \\
\hline 35.86 & 2.504 & 0.1667 & & & & & & & 34.84 & 2.575 & 0.1777 \\
\hline 35.87 & 2.503 & 1.393 & & & & & & & 35.78 & 2.510 & 0.1666 \\
\hline 36.55 & 2.458 & 0.0787 & & & & & & & 36.87 & 2.438 & 0.1444 \\
\hline 38.12 & 2.361 & 0.0515 & & & & & & & 37.32 & 2.409 & 0.1166 \\
\hline 42.46 & 2.129 & 0.0939 & & & & & & & 39.85 & 2.262 & 0.2222 \\
\hline 43.21 & 2.094 & 0.1333 & & & & & & & 41.36 & 2.183 & 0.0527 \\
\hline 44.18 & 2.050 & 0.1288 & & & & & & & 43.67 & 2.073 & 0.1666 \\
\hline 47.45 & 1.916 & 0.1060 & & & & & & & 46.43 & 1.956 & 0.1611 \\
\hline 48.27 & 1.885 & 0.0303 & & & & & & & 47.94 & 1.898 & 0.1388 \\
\hline 49.08 & 1.856 & 0.0606 & & & & & & & & & \\
\hline
\end{tabular}

absolutely new individual compounds are obtained which are in full conformity with the results of the above given micro elemental analysis and melting point measurements (Tables 1 and 2).

$\mathrm{X}$-ray diffraction patterns of the composites $\mathrm{Fe}_{2} \cdot \mathrm{Mn} \cdot \mathrm{L}_{2} \cdot \mathrm{H}_{2} \mathrm{O}$ and $\mathrm{Fe}_{2} \cdot \mathrm{Co} \cdot \mathrm{L}_{2} \cdot 4 \mathrm{H}_{2} \mathrm{O}$ are characterized by weakly expressed diffuse peaks, therefore they represent X-ray-amorphous compounds. Other compounds are characterized by clearly expressed crystalline structures, various relative intensities $\left(I / I_{0}\right)$, reflection angles $(2 \theta)$, and definite intra-spatial distance $(d)$ values. It should be stated that X-ray diffraction patterns of the composites $\mathrm{Cu}_{2} \cdot \mathrm{Co} \cdot \mathrm{L}_{2} \cdot \mathrm{H}_{2} \mathrm{O}$ and $\mathrm{Co}_{2} \cdot \mathrm{Cu} \cdot \mathrm{L}_{2} \cdot 4 \mathrm{H}_{2} \mathrm{O}$ are identical, which speaks of their iso-structure that is conditioned by analogousness of their chemical composition and geometrical characteristics of crystalline structure, which can not be said about the composites: $\mathrm{Zn}_{2} \cdot \mathrm{Mn} \cdot \mathrm{L}_{2} \cdot 2 \mathrm{H}_{2} \mathrm{O}, \quad \mathrm{Co}_{2} \cdot \mathrm{Zn} \cdot \mathrm{L}_{2}, \quad \mathrm{Co}_{2} \cdot \mathrm{Mn} \cdot \mathrm{L}_{2} \cdot 4 \mathrm{H}_{2} \mathrm{O}$, $\mathrm{Mn}_{2} \cdot \mathrm{Cu} \cdot \mathrm{L}_{2} \cdot 4 \mathrm{H}_{2} \mathrm{O}, \mathrm{Fe}_{2} \cdot \mathrm{Zn} \cdot \mathrm{L}_{2} \cdot 2 \mathrm{H}_{2} \mathrm{O}$ (Table 3). 


\section{Conclusions}

For the first time, we synthesized Heteronuclear chelate citrates of general formula: $\mathrm{M}_{2}^{\mathrm{I}} \mathrm{M}^{\mathrm{II}} \mathrm{L}_{2} \cdot n \mathrm{H}_{2} \mathrm{O}$ by using modified and simplified methods. We studied identity and composition of synthesized compounds, determined their crystallinity, and revealed X-ray amorphous and iso-structural orders.

We have conducted preliminary studies on these synthesized compounds as new poultry premixes. Based on good preliminary data, we concluded to conduct large-scale studies in this direction.

\section{Acknowledgments}

We thank Science \& Technology Center in Ukraine and ShotaRustaveli National Science Foundation. The work was implemented with the support of Science \& Technology Center in Ukraine Project Proposal \#5461 and ShotaRustaveli National Science Foundation Grant \#30/06.

\section{References}

[1] Tanatarov, A. B. Mineral Elements in Biology and Their Use in Medicine and Agriculture. Moscow 1986, 3, 358.

[2] Kalashnikov, A. P.; Fisinin, V. I.; Scheglev, V. V. Norms and Rations in the Feeding of Agricultural Animals; Moscow, 2003; p 456.

[3] Menkin, V. K. The Feeding of Animals; Moscow, 2003; p 360 .

[4] Draganov, I. F.; Buryakova, M. A. Working Program of Teaching Discipline "Essentials of Research in Agricultural Animals”; Timiryazeva, 2006; p 13.

[5] Dobrinina, N. A. Biological Chemistry; Lomonosov Moscow State University: Moscow, 2007; p 36.

[6] Ribkina, T. I. Biological and Toxic Impact of Chemical Elements and Their Inorganic Compounds on Human
Organism; Novomoskovsk, 1999; p 92.

[7] Dobrinina, N. A. Bioinorganic Chemistry; Moscow, 2007; p 36.

[8] Lebedev, S. V.; Miroshnikov, S. A.; Sukhanova, O. N.; Rakhmatullin, S. G.; Malyishin, E. N.; Sipailova, O. Y.; et al. Method for Increase of Productivity of Chicken-Broilers. Patent 2,370,095, Oct. 20, 2010.

[9] Beshkenadze, I. A.; Tsitsishvili, V. G.; Urotadze, S. L.; Gogaladze, M. A.; Zhorzholiani, N. B. In Natural Zeolites and Biometal-Containing Compozites, Proceedings of Materials of IV International Conference Sorbents as Factors of Life Quality and Health, Belgorod, Sept 24-28, 2012.

[10] Beshkenadze, I. A.; Gogaladze, M. A.; Zhorzholiani, N. B.; Urotadze, S. L.; Burkiashvili, N. O.; Gogua, L. D. Synthesis of the Chelates Containing Amino Acids and Citric Acid for Creation of New Generation Premixes. Annals of Agrarian Science 2013, 11(2), 84-86.

[11] Beshkenadze, I.; Gogaladze, M.; Zhorzholiani, N. Chemical Admix to Poultry Fodder; National Center for Intellectual Property of Georgia: Tbilisi, 2000.

[12] Beshkenadze, I.; Gogaladze, M.; Zhorzholiani, N. Chemical Admix to Poultry Fodder; National Center for Intellectual Property of Georgia: Tbilisi, \#P4917, 2008.

[13] Beshkenadze I., Zhorzholiani N., Gogaladze M. Chemical Admix to Poultry Fodder; National Center for Intellectual Property of Georgia: Tbilisi, \#P4918, 2008.

[14] Beshkenadze, I.; Zhorzholiani, N.; Gogaladze, M. Chelate-Containing Chemical Admix for Poultry Feeding; 2013.

[15] Kozmanishvili, A.; Beshkenadze, I.; Zhorzholiani, N. Chemical Editions for Rabbit Food Ration; Bulletin of the Academy of Agricultural Sciences of Georgia: Tbilisi 2003.

[16] Beshkenadze, I.; Gogaladze, M.; Zhorzholiani, N. Synthesis of the Chelates Continuing Amino Acids and Citric Acid for Creation of New Generation Premixes. Annals of Agrarian Science 2013, 11(2), 84-86.

[17] Tsintsadze, G.; Beshkenadze, I.; Mestiashvili, N.; Zhorzholiani, N. In Physical-Chemical Investigation of $M^{I} M^{I I} L \cdot n H_{2} \mathrm{O}$ Heteronuclear Citrates, Proceedings of the Georgian Academy of Sciences, 2006. 